\title{
Back to basics in ultrasound velocimetry: tracking speckles by using a standard PIV algorithm
}

\author{
Vincent Perrot*, Damien Garcia* \\ *Univ. Lyon, INSA-Lyon, UCBL, UJM-Saint-Étienne, CNRS, Inserm, CREATIS UMR 5220, U1206, Lyon, France \\ vincent.perrot@creatis.insa-lyon.fr
}

\begin{abstract}
In this IUS proceeding, we describe a classical block-matching approach that we used during the 2018 SA-VFI (Synthetic Aperture 2-D Vector Flow imaging) challenge. To estimate frame-to-frame displacements, we used blockwise FFT-based ensemble cross-correlations. Subpixel displacements were obtained by parabolic peak fitting. We opted for a coarse-to-fine multiscale scheme to increase the resolution and precision. Robustness and accuracy were improved by including a robust unsupervised smoother in the estimation process. 2-D velocity vector fields were computed in several flow phantoms (from both simulations and experiments) provided by the organizers of the SA-VFI challenge. Our results showed that the standard block-matching approach provided reliable 2-D velocity vector fields, in terms of magnitude and angle. Complex flow patterns, like those occurring in the carotid bifurcation, were also estimated accurately. In summary, the long-standing block-matching technique by normalized cross-correlation is effective for flow estimation by ultrasound imaging. The final estimates returned by the method will be uploaded on the SA-VFI platform, and the results will be compared with those obtained by all the competitors during the challenge session at IEEE IUS 2018 in Kobe (Japan).
\end{abstract}

Index Terms-Speckle tracking, multiscale block-matching, normalized cross-correlation, unsupervised smoothing, flow estimation, synthetic aperture

\section{INTRODUCTION}

Particle imaging velocimetry (PIV) is an optical method of flow visualization that originated from laser speckle velocimetry in the 1980s [1]. This technique has been extended to ultrasound in the 1990s [2] for velocity vector imaging of the blood circulation. It is generally called "echo-PIV" when blood is seeded with contrast agents [3]. If an appropriate wall filter is used, echo-PIV can be achieved by tracking the natural speckle patterns issued from the blood scatterers [4]. Echo-PIV has been used to investigate the complex flow patterns that can occur in the cardiovascular system [4], [5], especially in the left ventricular cavity.

In 2018, Jensen et al. organized an ultrasound flow-imaging challenge entitled "SA-VFI (synthetic aperture 2-D vector

This work was partially funded thanks to the GdR ISIS. The Verasonics system was co-funded by the FEDER program, Saint-Etienne Metropole (SME) and Conseil General de la Loire (CG42) within the SonoCardioProtection Project headed by Prof. Pierre Croisille and Dr. Magalie Viallon, principal investigators. This work has been performed within the framework of the LABEX CELYA (ANR-10-LABX-0060) and LABEX PRIMES (ANR-10-LABX-0063) of Université de Lyon, within the program "Investissements d'Avenir" (ANR-11-IDEX-0007) operated by the French National Research Agency (ANR). velocity imaging) challenge", as part of the 2018 International Ultrasonics Symposium (IUS) at Kobe, Japan [6]. Synthetic aperture is a parallel imaging technique that consists in forming an image by transmitting a number of wide wavefronts using different sub-array apertures. The objective of the SA-VFI challenge was to estimate blood flow velocities in some datasets issued from simulations and in vitro experiments. The participants were asked to test their algorithms in a training dataset before being evaluated on a blinded evaluation dataset.

Section II introduces the datasets and the echo-PIV method that we used for velocity vector estimation. Section III presents some results returned by echo-PIV. Finally, section IV discusses the potential applications of the presented approach.

\section{MATERIAL AND METHODS}

\section{A. Datasets}

Acquisitions were performed using a synthetic aperture approach in both simulations and in vitro experiments [6]. A total of six datasets were provided by the SA-VFI organizers: 1) a simulated spinning disk; 2) a simulated and 3) an experimental straight vessel at $90^{\circ}$;4) a simulated and 5) an experimental straight vessel at $105^{\circ}$;6) and a simulated carotid bifurcation model [7]. Acoustic waves were transmitted through 64 adjacent elements, by using five distinct virtual sources located behind the probe. A full aperture (128 elements) was used during reception. The transmit and receive parameters are described in Table I; the reader can refer to [6] for more information.

TABLE I

ACQUISITION PARAMETERS FOR THE FLOW SEQUENCE

\begin{tabular}{|c|c|}
\hline Parameter & Value \\
\hline \hline Center frequency & $8 \mathrm{MHz}$ \\
\hline Sampling frequency & $35 \mathrm{MHz}$ \\
\hline Number of elements & 128 \\
\hline Pitch & $300 \mu \mathrm{m}$ \\
\hline Elevation focus & $20 \mathrm{~mm}$ \\
\hline Transmit pulse & $\begin{array}{c}\text { 3-cycle sinusoidal pulse } \\
\text { with a 50\% Tukey window }\end{array}$ \\
\hline Speed of sound & $1480-1540 \mathrm{~m} / \mathrm{s}$ \\
\hline Pulse repetition frequency & $5000-15000 \mathrm{~Hz}$ \\
\hline Number of emitting elements & 64 \\
\hline Number of receiving elements & 128 \\
\hline F-number & -3.5 \\
\hline Distinct beams & 5 \\
\hline
\end{tabular}




\section{B. Beamforming}

The signals were beamformed using a standard diffraction summation (delay-and-sum, DAS) on a fine grid after I/Q demodulation with respect to the center frequency $(8 \mathrm{MHz})$. The delay due to the length of the transmit pulse convolved with the 2-way impulse was compensated to obtain a correct match between the envelope of the beamformed data and the scatterers. We tested different beamforming parameters, as provided in Table II. The final parameters were chosen based on the velocity estimates. In Table II, the bold text indicates the settings that we selected for the SA-VFI challenge.

TABLE II

BEAMFORMING SET-UP

\begin{tabular}{|c|c|}
\hline Parameter & Value \\
\hline \hline F-number & $1.0 ; \mathbf{1 . 5} ; 3.0$ \\
\hline & Rectangular \\
Received apodization & Tukey $25 \%$ \\
& Tukey $\mathbf{5 0 \%}$ \\
& Hann \\
\hline Compounding & Yes; No \\
\hline Grid resolution (regular) & $\lambda ; \lambda / 2 ; \boldsymbol{\lambda} / \mathbf{4} ; \lambda / 8$ \\
\hline
\end{tabular}

\section{Speckle tracking algorithm}

Before speckle tracking, a $1^{\text {st }}$ order Butterworth high-pass filter with a cutoff velocity at $1 \mathrm{~cm} / \mathrm{s}$ was applied on the signals to remove stationary echoes. Speckle tracking was implemented using a standard FFT-based phase correlation on the envelopes of the delay-and-summed I/Q signals (Fig. 1) [8].

The technique can be summarized as follows: two successive real-envelope images \#1 and \#2 are subdivided into $(\mathrm{m} \times \mathrm{n})$ windows. Let $w_{1}^{k}$ and $w_{2}^{k}$ be the kth subwindows (Fig. 1). Using their respective Fourier transforms $W_{1}^{k}$ and $W_{2}^{k}$, the normalized FFT-based normalized cross-correlation (NCC) is given by

$$
N C C^{k}=\mathscr{F}^{-1}\left(\frac{W_{1}^{k} \overline{W_{2}^{k}}}{\left|W_{1}^{k} W_{2}^{k}\right|}\right)
$$

The operator $\mathscr{F}^{-1}$ stands for the inverse Fourier transform. The relative displacements $\left(\Delta_{i}^{k}, \Delta_{j}^{k}\right)$ into a grid $(i, j)$, from the subwindows $w_{1}^{k}$ to the subwindows $w_{2}^{k}$, can be obtained by determining the position of the NCC peak:

$$
\left(\Delta_{i}^{k}, \Delta_{j}^{k}\right)=\arg \max _{(i, j)}\left(N C C^{k}\right)
$$

To obtain subpixel displacements, we used a parabolic peak fitting around the NCC peak. The actual movements (in $\mathrm{mm}$ ) were recovered by knowing the pixel size. To detect both large and small displacements, we opted for a coarse-to-fine multiscale approach: the displacement estimates were refined iteratively by decreasing the size of the subwindows. This prediction-correction scheme led to improved resolution and precision. In this SA-VFI challenge, we used a four-scale subwindowing. The estimated displacements were smoothed, with a robust unsupervised spline smoother, between two consecutive scales [9].

During the SA-VFI challenge, this algorithm was used with an ensemble length of 35 received acquisitions (7 frames $\times 5$ distinct beams) for integration in the normalized cross-correlation (ensemble cross-correlation). Several parameters were explored for speckle tracking by cross-correlation, as provided in Table III. The bold text refers to the values that we chose in the final set-up. After speckle

TABLE III

\begin{tabular}{|c|c|}
\hline Parameter & Value \\
\hline $\begin{array}{l}\text { Consecutive window sizes } \\
\qquad(\mathrm{mm})\end{array}$ & $\begin{array}{c}4 ; 3 ; 2 ; 1 \\
\mathbf{4} ; \mathbf{2 . 5} \mathbf{2} ; \mathbf{1} \\
4 ; 2 ; 1 ; 0.5 \\
3 ; 1.5 ; 1 ; 0.5 \\
2.5 ; 1.5 ; 1 ; 0.5 \\
2 ; 1 ; 0.5 ; 0.25\end{array}$ \\
\hline Subwindow overlap & $\begin{array}{c}50 \% ; 55 \% ; 60 \% ; \mathbf{6 5 \%} \\
70 \% ; 75 \% ; 80 \%\end{array}$ \\
\hline $\begin{array}{c}\text { Ensemble length } \\
(\text { frames } \times 5 \text { distinct beams })\end{array}$ & $\begin{array}{l}5 ; 15 ; 25 ; 35 ; 45 ; 55 ; 65 \\
75 ; 85 ; 95 ; 105 ; 115 ; 125\end{array}$ \\
\hline
\end{tabular}

SPECKLE TRACKING SET-UP

tracking, the 2-D velocity vector fields were interpolated onto the grid provided by the organizers of the SA-VFI challenge.

\section{RESUlTS}

The evaluation metrics were computed through the SA-VFI platform from the velocity fields estimated by our approach. We here present those obtained with the spinning disk (Fig. 2) and the simulated straight vessel at $105^{\circ}$ (Fig. 3). The results show that the PIV method can provide accurate velocity estimates in terms of both magnitude and angle. Moreover, the standard deviations were relatively low with the two datasets (Fig. 2 and Fig. 3), which indicates that the method has acceptable precision. Indeed, for the spinning disk, the relative bias and standard deviation of the velocity magnitude were $6.26 \%$ and $1.93 \%$, respectively. Regarding the angle estimates, the bias was $0.95^{\circ}$ and the standard deviation was $0.97^{\circ}$. The vessel tests also revealed accurate measurements, with $0.78 \% / 0.02^{\circ}$ and $1.32 \% / 1.23 \%$ for the velocity / angle magnitude bias and standard deviation, respectively. For the sake of illustration, 2-D velocity vector fields were extracted from the carotid bifurcation model (Fig. 4) during systole. This dataset presents a more complex flow pattern than those of the spinning disk and the straight vessels. The multiscale speckle tracking PIV algorithm allowed us to recover the blood flow pattern successfully (Fig. 4).

\section{CONCLUSION AND DISCUSSION}

In this proceeding, we presented the standard approach that we used during the SA-VFI challenge. The technique that we tested was based on block-matching by cross-correlation with a multiscale scheme and a robust smoothing procedure.

Our results show that complex laminar flow patterns, at both low and high flow rates, can be deciphered accurately by a 


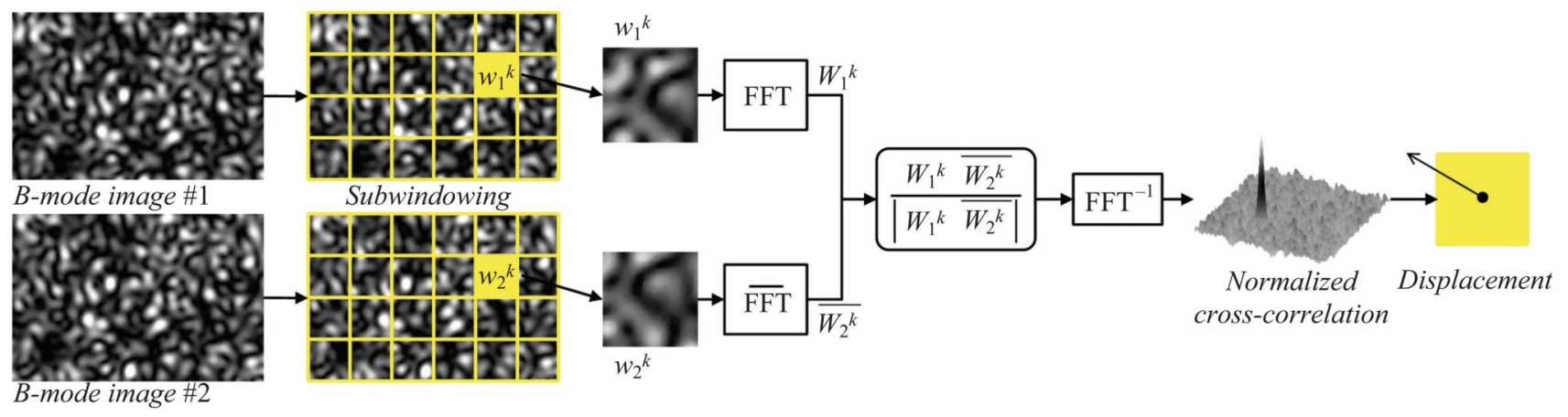

Fig. 1. Speckle tracking algorithm implemented in the Fourier domain based on the phase correlation; from [8] with permission.
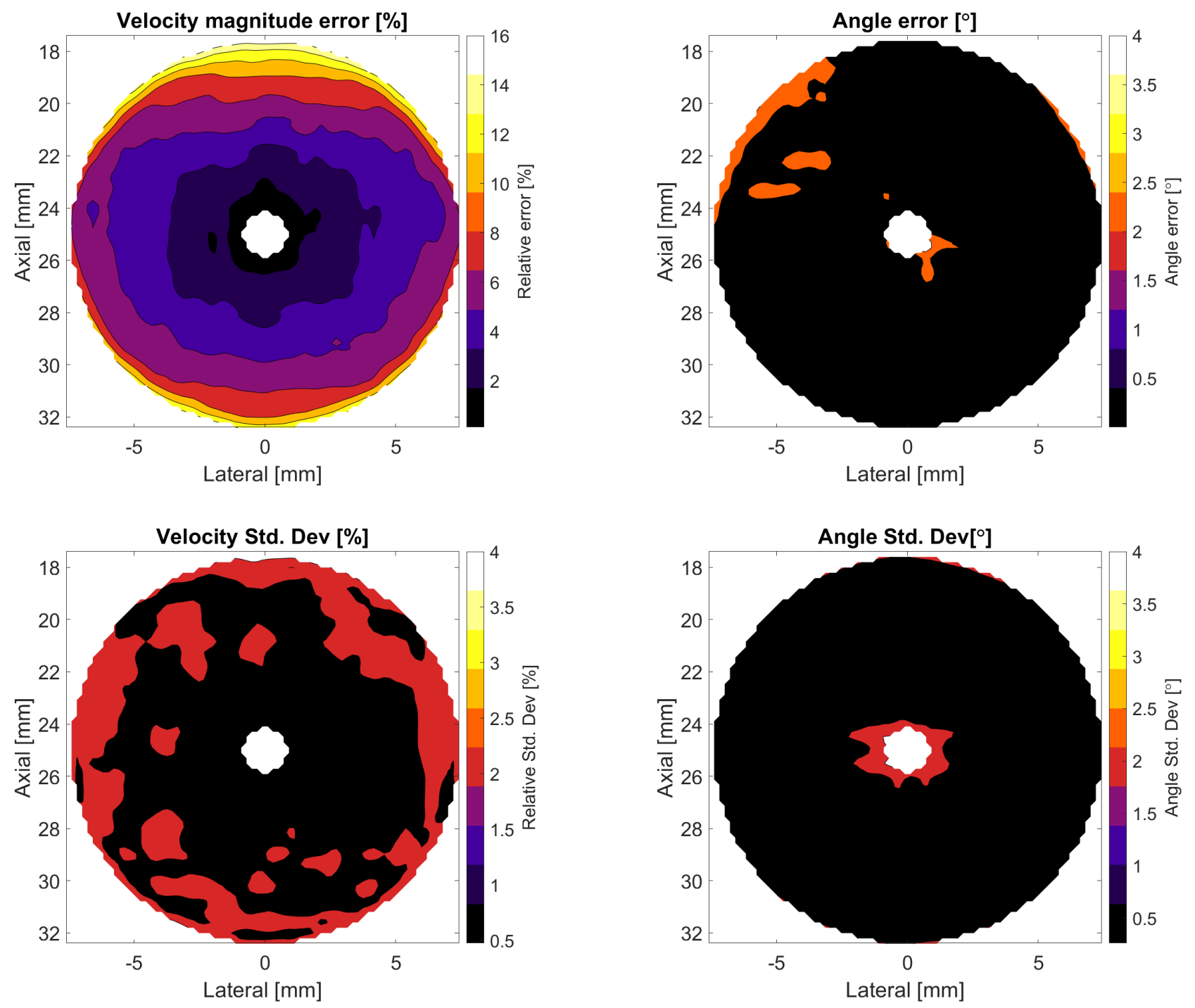

Fig. 2. Metrics computed for the simulated disk: (top-left) magnitude error in percentage, (top-right) angle error in degree, (bottom-left) standard deviation of the estimated velocity in percentage and (bottom-right) standard deviation of the estimated angle in degree. 

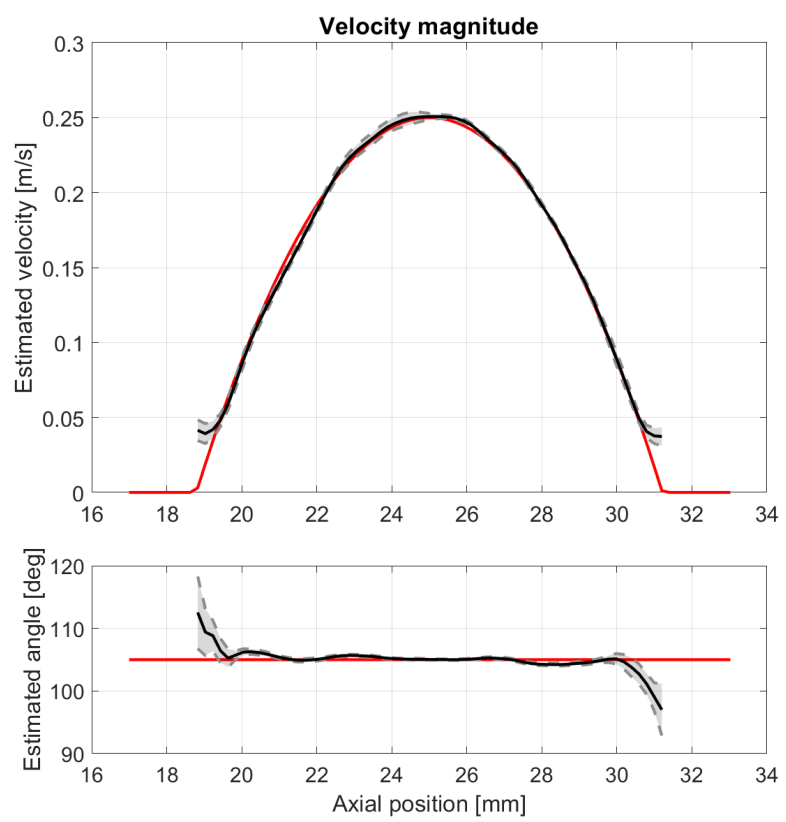

Fig. 3. Metrics computed for the simulated $105^{\circ}$ vessel: (top) velocity magnitude error in percentage and (bottom) estimated angle.

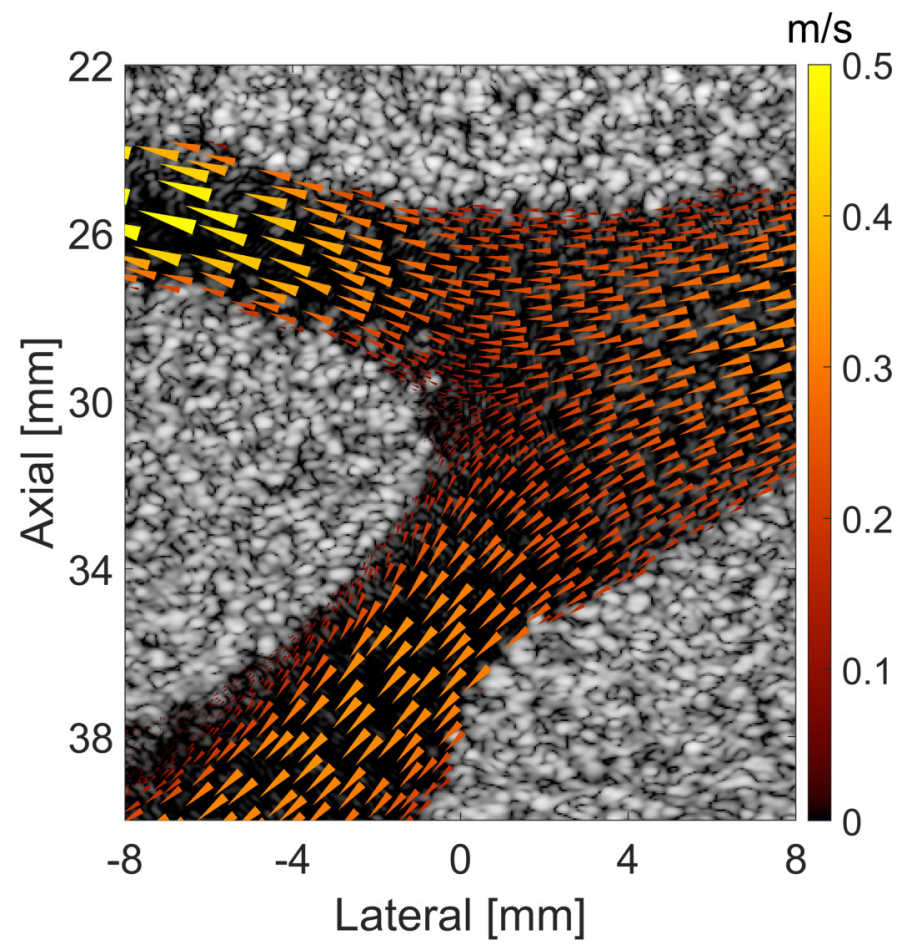

Fig. 4. B-mode image superimposed with the flow estimate for the carotid bifurcation during systole. standard echo-PIV algorithm. This algorithm is commonly used in research for estimating tissue and blood motions. A multiscale scheme should be mandatory to ensure covering the wide velocity ranges observed in the vascular system. It seemed that "the keep it simple" motto was a fair practice in this SA-VFI challenge. According to the narrow error ranges that we obtained, however, it is noteworthy that the simulation models were likely not sufficiently discriminating and therefore unsuitable for a clear-cut comparison of different flow-tracking techniques. We also noticed that the smoother had a significant impact on the outputs. At this stage, however, we cannot judge which of the tracker or the smoother was the most impactful on the accuracy of the velocity estimates. As a final note, we had no control over the transmit sequences, which makes it impossible to test how vector Doppler by plane wave imaging [10] compares with other techniques. At the completion of this challenge, any hasty conclusion on the respective performance of the different methods should be avoided, since the actual physiological world can drastically change the situations.

\section{REFERENCES}

[1] C. E. Willert and M. Gharib, "Digital particle image velocimetry," Experiments in Fluids, vol. 10, no. 4, pp. 181-193, jan 1991.

[2] G. Trahey, S. Hubbard, and O. von Ramm, "Angle independent ultrasonic blood flow detection by frame-to-frame correlation of b-mode images," Ultrasonics, vol. 26, no. 5, pp. 271-276, sep 1988.

[3] H. B. Kim, J. R. Hertzberg, and R. Shandas, "Development and validation of echo PIV," Experiments in Fluids, vol. 36, no. 3, pp. 455-462, mar 2004

[4] S. Fadnes, S. A. Nyrnes, H. Torp, and L. Lovstakken, "Shunt flow evaluation in congenital heart disease based on two-dimensional speckle tracking," Ultrasound in Medicine \& Biology, vol. 40, no. 10, pp. 2379-2391, oct 2014

[5] G.-R. Hong, G. Pedrizzetti, G. Tonti, P. Li, Z. Wei, J. K. Kim, A. Baweja, S. Liu, N. Chung, H. Houle, J. Narula, and M. A. Vannan, "Characterization and quantification of vortex flow in the human left ventricle by contrast echocardiography using vector particle image velocimetry," JACC: Cardiovascular Imaging, vol. 1, no. 6, pp. 705-717, nov 2008.

[6] J. A. Jensen, H. Liebgott, F. Cervenansky, and C. A. V. Hoyos, "SA-VFI: the IEEE IUS challenge on synthetic aperture vector flow imaging," in 2018 IEEE International Ultrasonics Symposium (IUS). IEEE, 2018.

[7] A. Swillens, J. Degroote, J. Vierendeels, L. Lovstakken, and P. Segers, "A simulation environment for validating ultrasonic blood flow and vessel wall imaging based on fluid-structure interaction simulations: Ultrasonic assessment of arterial distension and wall shear rate," Medical Physics, vol. 37, no. 8, pp. 4318-4330, jul 2010.

[8] D. Garcia, "Introduction to speckle tracking in cardiac ultrasound imaging," in Handbook of Speckle Filtering and Tracking in Cardiovascular Ultrasound Imaging and Video. Institution of Engineering and Technology, 2018, pp. 571-598.

[9] —, "A fast all-in-one method for automated post-processing of PIV data," Experiments in Fluids, vol. 50, no. 5, pp. 1247-1259, oct 2010. [Online]. Available: http://www.biomecardio.com

[10] C. Madiena, J. Faurie, J. Poree, and D. Garcia, "Color and vector flow imaging in parallel ultrasound with sub-nyquist sampling," IEEE Transactions on Ultrasonics, Ferroelectrics, and Frequency Control, vol. 65, no. 5, pp. 795-802, may 2018. 